\title{
Systematic review and meta-analysis on perioperative intervention to prevent postoperative atelectasis complications after thoracic surgery
}

\author{
Yongsheng Zhao ${ }^{1}$, Renyan Zheng ${ }^{2}$, Wanping Xiang', Dong Ning ${ }^{1}$, Zhenglong Li ${ }^{1}$ \\ ${ }^{1}$ Department of Thoracic Surgery, Affiliated Hospital of North Sichuan Medical College, Nanchong, China; ${ }^{2}$ Department of Integrated Western and \\ Chinese Colorectal and Anal Surgery, Affiliated Hospital of North Sichuan Medical College, Nanchong, China \\ Contributions: (I) Conception and design: Y Zhao, R Zheng; (II) Administrative support: W Xiang; (III) Provision of study materials or patients: Y \\ Zhao, R Zheng, D Ning, Z Li; (IV) Collection and assembly of data: All authors; (V) Data analysis and interpretation: Y Zhao, W Xiang, D Ning, Z \\ Li; (VI) Manuscript writing: All authors; (VII) Final approval of manuscript: All authors. \\ Correspondence to: Yongsheng Zhao. Department of Thoracic Surgery, Affiliated Hospital of North Sichuan Medical College, 1 Maoyuan South Road, \\ Shunqing District, Nanchong 637000, China. Email: Yongsheng_zhao2021@126.com.
}

\begin{abstract}
Background: In clinical general thoracic surgery, the prevalence of atelectasis is relatively high. Perioperative interventions can affect the probability of patients with atelectasis after surgery. Therefore, the incidence of perioperative intervention to prevent atelectasis after thoracic surgery was discussed using metaanalysis in this study.

Methods: The articles were searched in the English database PubMed and Chinese databases including China National Knowledge Infrastructure (CNKI), VIP, and China Journal Full-text Database (CJFD). The duration for publication time of the articles was from the database inception to March 2021, and the articles were required to be randomized controlled trials (RCTs) using interventions [such as changing the dose of general anesthesia, continuous positive end expiratory pressure (PEEP), non-invasive pressure support ventilation, and physical therapy] after thoracic surgery (such as pulmonary lobectomy, sternum surgery, and lung cancer surgery) for the treatment of atelectasis. The software RevMan 5.3 provided by the Cochrane Collaboration was used for meta-analysis.

Results: A total of 5 articles were obtained, including 375 cases in the control group and 268 cases in the intervention treatment group. A meta-analysis was performed on the included articles, combined effect model analysis results showed that compared with the control group, the use of PEEP during mechanical ventilation can significantly reduce the incidence of atelectasis [odds ratio $(\mathrm{OR})=0.46$; $95 \%$ confidence interval (CI): 0.31-0.67; $\mathrm{Z}=3.94 ; \mathrm{P}<0.0001]$.
\end{abstract}

Discussion: Perioperative intervention was more effective for postoperative atelectasis and other complications.

Keywords: Thoracic surgery; perioperative period; meta-analysis; atelectasis

Submitted Aug 12, 2021. Accepted for publication Oct 13, 2021.

doi: $10.21037 /$ apm-21-2441

View this article at: https://dx.doi.org/10.21037/apm-21-2441

\section{Introduction}

The prevalence of atelectasis accounts for $30.00-75.00 \%$ of ordinary thoracic surgery, of which $20.00-42.00 \%$ is due to pulmonary infections after surgery, and the mortality rate is as high as $27.00-50.00 \%(1,2)$. The treatment of atelectasis and pulmonary infection after thoracic surgery has great clinical value. Although progress has been made in the perioperative care of patients undergoing major surgery, postoperative pulmonary complications (PPCs) are the main cause of morbidity and mortality. The grouping of 
PPC includes a series of diseases that affect the respiratory system, usually alveolar expansion or respiratory failure in the first week after surgery. The physical condition of the patient is also related to the success of the operation. Atelectasis, pneumonia, tracheobronchitis, bronchospasm, acute exacerbation of chronic obstructive pulmonary disease, hypoxemia, dyspnea, acute respiratory failure, and difficulty in weaning can all be diagnosed as pulmonary complications after thoracic surgery. The main causes are postoperative respiratory muscle dysfunction, ventilatory blood flow ratio imbalance, central and peripheral nervous system depression, reduced cough efficiency, respiratory secretions retention, increased respiratory frequency, decreased tidal volume, functional residual capacity, and vital capacity. Postoperative pain is related to analgesia. For example, performing ankle surgery on healthy young people may have a less than $1 \%$ risk, while performing surgery on frail elderly patients bears a higher risk. When thoracic surgery is performed, the surgical incision is close to the thoracic cavity, which increases the risk of infection. Complications can cause pain, which will stymie the muscle recovery after surgery. Age is one of the predictive indicators of PPC, evidenced by the fact that healthy elderly patients are at higher risk of PPC (3). The PPCs are also predictors of short- and long-term recovery after surgery, and the increased risk of patients receiving critical care is also related to prolonged hospital stay $(4,5)$. About $14-30 \%$ of PPC patients may die within 30 days after surgery, while the mortality rate of patients without PPC is $0.2-3 \%$. The causes of PPCs are multi-factorial and are related to the patient's suboptimal health status and the acute side effects of surgery accompanied by anesthesia $(6,7)$. The surgery itself can inhibit lung function, especially when the operation is severe enough to impair breathing. Anesthesia has an adverse effect on lung function during the operation, which may continue to a lesser extent until the recovery time after the operation. Chronic risk factors for PPC include poor cardiorespiratory health, increasing age, lifestyle, and living habits $(8,9)$.

Fortunately, there are multiple opportunities for intervention, which may hinder the development of PPC. Interventions are diverse, including preemptive strategies (before surgery) to optimize respiratory physiology, and intraoperative and postoperative interventions to minimize the adverse effects of surgery and anesthesia (10). The treatment of PPCs requires the multidisciplinary participation of anesthesia, surgery, respiratory medicine, physical therapy, and intensive care professionals, and bears the burden of related economic and health outcomes $(11,12)$. Nevertheless, compared with postoperative cardiovascular complications, consensus guidelines for perioperative management to reduce the risk of PPCs are still rare. Due to the extensive and diverse evidence base of many interventions and the lack of consensus, there are great differences in clinical practice $(13,14)$.

The objective of this systematic review was to summarize the evidence of randomized controlled trials (RCTs), with the aim of reducing PPC after thoracic surgery in adults. This study aimed to compare the quality, quantity, and deviation risk of PPC management treatment effect. The inherent focus of this approach was to focus on whether the benefits are related to each treatment, rather than comparing their side effects. This was because although the benefits of treatment should be similar, the harms are very different due to them working very differently. In this study, 8 articles related to the treatment of postoperative pulmonary infections and the treatment effect of atelectasis after thoracic surgery were included for multiple screening and meta-analysis on results of domestic and international comparative studies of cases. It aimed to provide a scientific and theoretical basis for postoperative pulmonary infection and prevention of atelectasis after thoracic surgery. We present the following article in accordance with the PRISMA reporting checklist (available at https://dx.doi. org/10.21037/apm-21-2441).

\section{Methods}

\section{Strategy for article retrieval}

The databases of Medline, Embase, Cumulative Index of Nursing and Allied Health Literature (CINHAL), and Cochrane Central Register of Controlled Trials were searched by taking the related keywords and medical subject heading terms of PPCs. The researched articles were limited to RCTs published from 1 January 1990 to 8 December 2020 and RCTs related to contemporary surgical and anesthesia practices, including laparoscopic surgical techniques. According to the European Perioperative Clinical Outcome (EPCO) definition and combined with the descriptive terms (such as intraoperative complications, perioperative complications, preoperative care, intraoperative care, postoperative care, and anesthesiaassociated with postoperative respiratory complications, the Chinese search keywords included atelectasis, postoperative complications, postoperative atelectasis, 
thoracic surgery, pulmonary lobectomy, sternotomy, lung cancer intervention, general anesthesia, and physical intervention. The full text of the target articles could be obtained in accordance with the pre-established inclusion and exclusion criteria. In addition, the perioperative fluid and hemodynamic management strategies, intraoperative neuromuscular block and monitoring, airway devices, and lung aspiration techniques were studied.

\section{Inclusion and exclusion criteria of the articles}

The inclusion criteria were defined as follows: articles which were RCTs; articles which compared the incidence of pulmonary infection and atelectasis after thoracic surgery no matter with hidden or blind method; articles whose research results contained the relevant goals involved in this study; articles which investigated the vital signs of patients with thoracic surgery; and articles which introduced the treatment methods and processes of pulmonary infections and atelectasis.

The exclusion criteria were defined as follows: articles involving participants under 18 years old; articles with repeated data; articles with inconsistent data that could not be explained by reporting bias (data error); and articles including participants with a history of adverse reactions.

\section{Screening of articles}

The articles were screened and data was extracted independently, and cross-checking was performed. In the case of different opinions, expert opinions were sought to determine the data selection.

\section{Data extraction}

The data extraction of this study was carried out independently by two researchers. During data extraction, two researchers firstly extracted the data independently, and created an Excel table based on the basic information of the articles, participant characteristics, intervention measures, outcome indicators, and bias evaluation. After the extraction was completed, cross-check was performed. If there were differences of opinion during the data extraction process, the researchers sought resolution through discussion or solicited the opinion of a third researcher. The data to be extracted in this study included basic information of the article (title, first author, publication year, author information, source, and so on), basic participant characteristics (gender, age, research sample size, baseline comparability, and so on), research methods, research plan design, intervention measures for experimental group and control group, outcome evaluation indicators, and outcome data.

\section{Quality assessment}

To assess the quality of the articles, quality evaluation was carried out according to the "bias risk assessment" recommended by Cochrane system review manual (version 5.3; https://training.cochrane.org/handbook). The evaluation contents included the following 7 items: which random method was used; whether allocation concealment was used; implementation of blinding between patients and researchers; assessment on the effect of blinding; whether the results were complete; whether the survey results were credible; and other biases. Regarding the RCTs in item 7 above, "satisfied" meant that the bias was relatively small and "unsatisfied" meant a high degree of bias. If the study had not been fully reported in detail, the risk was deemed unknown. The modified Jadad scale review was used to assess the quality of the attached research and literature. The evaluation included random sequence generation, allocation concealment, blinding, and tracking/exit with a score of 1 to 3 , which was considered low quality, and 4 to 7 points were considered high quality.

\section{Lung ultrasound (LUS) score for diagnosis of atelectasis}

Diagnosis of atelectasis: divide the chest into 12 areas, both the left and right chests have 6 areas, each side is divided into anterior area, lateral area, posterior area by the parasternal line, anterior axillary line, posterior axillary line, and the nipple. The upper $1 \mathrm{~cm}$ is the boundary, which is divided into upper and lower areas. Ventilation loss is evaluated by calculating the LUS score. According to the scoring system, each of the 12 districts is rated as 0 to 3 points, and then the 12 quadrant scores are added together to calculate the total LUS score (0 to 36 points), and the higher score indicates that the ventilation loss is more serious. LUS scoring criteria for each area is as follows: 0 points for normal ventilation ( 0 to 2 lines $\mathrm{B}$ ), 1 point for mild ventilation loss (greater than or equal to 3 lines B), 2 points for moderate ventilation loss (poly B line), and 3 points for severe ventilation loss (consolidation).

\section{Statistical analysis}

The RevMan 5.3 software (Copenhagen: The Nordic 


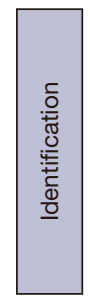

Identification of studies via databases and registers
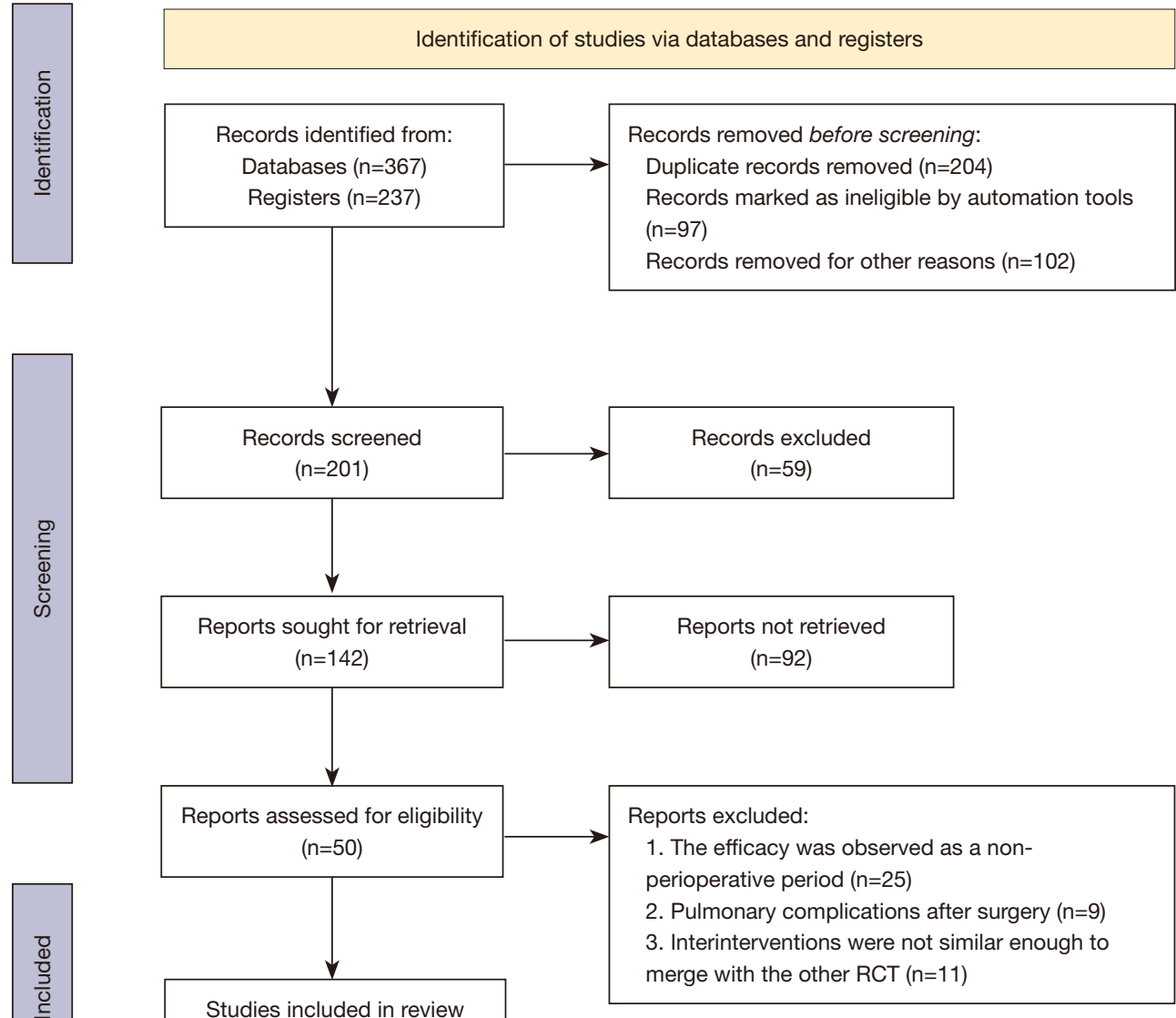

$(n=5)$

Figure 1 The flow chart for the article retrieval process. RCT, randomized controlled trial.

Cochrane Center, The Cochrane Collaboration, 2014) was used for meta-analysis. The calculation method took odds ratio (OR) as the effect size, and $95 \%$ confidence interval (CI) to express the result. A heterogeneity test was performed on the included articles, and $\alpha=0.1$ was taken as the test level. If there was no heterogeneity among the articles $\left(\mathrm{P}>0.1 ; \mathrm{I}^{2}<50 \%\right)$, the fixed effects model (FEM) was selected for meta-analysis; otherwise, subgroup analysis was performed on the included data. A $\mathrm{P}$ value $<0.05$ indicated that the difference was statistically significant.

\section{Results}

\section{Results of articles retrieval}

Initially, a total of 604 articles were retrieved. After reading the title of the article, 403 literatures that did not fit the research theme were initially excluded. Then, we briefly browsed the abstracts of the literature, and eliminated 59 literatures that did not meet the inclusion criteria. After carefully reading the titles and abstracts of the documents, 92 literatures that did not meet the requirements were further eliminated. After downloading the literature, read the specific content of the literature, and delete 25 literatures on "pulmonary complications after surgery", 9 literatures on "pulmonary complications after surgery", 11 literatures on "interinterventions were not similar enough to merge with the other RCT". Finally, a total of 5 documents meeting the requirements were included for meta-analysis (14-18). The literature retrieval process was shown in Figure 1. Funnel chart analysis showed that there was no obvious evidence of publication bias, or that the results of the smaller trial were systematically different from the larger trial. According to Cochrane criteria, the risk of bias in most articles could be judged. The basic characteristics of the document were shown in Table 1 . 
Table 1 The basic characteristics of the included articles

\begin{tabular}{|c|c|c|c|c|c|}
\hline Author & Publication year & Intervention method & Age (years old) & Number of cases & Study methoo \\
\hline Futier (15) & 2013 & Continuous PEEP & Unclear & 400 & $\mathrm{RCT}$ \\
\hline Talab (16) & 2009 & Non-invasive pressure support ventilation & Unclear & 66 & $\mathrm{RCT}$ \\
\hline Park (17) & 2016 & Physical therapy & $\geq 18$ & 40 & $\mathrm{RCT}$ \\
\hline
\end{tabular}

PEEP, positive end expiratory pressure; RCT, randomized controlled trial.

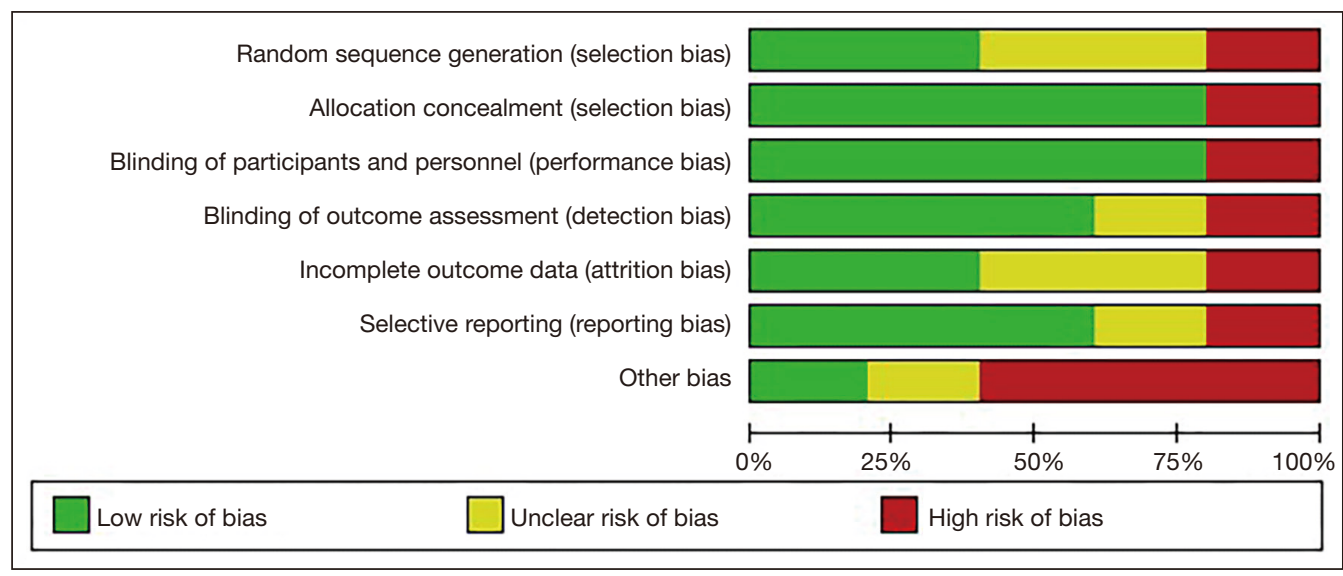

Figure 2 Assessment of risk bias of the included articles.

\section{Risk bias assessment of the included articles}

Cochrane Handbook 5.3 version of the systematic review writing manual was adopted to evaluate the risk of bias in the 5 articles included in this study and output the risk of bias chart, as shown in Figures 2,3. The risk of bias included the following items: (I) whether it was a random sequence: 2 of the 5 included articles reported the random grouping, suggesting that these 2 articles were all in low risk. Two article did not report whether it was random grouping, suggesting that these were unclear risk; and 1 article clearly mentioned the grouping method as "non-random", suggesting that it was high risk; (II) mention of allocation concealment: 4 articles mentioned allocation concealment, indicating that these 4 articles were in low risk, and 1 article did not mention whether allocation concealment was used, suggesting that is was high risk; (III) whether to blind participants: 1 article mentioned the patient's informed consent, indicating high risk, and other articles were low risk; (IV) whether to blind the outcome evaluators: 3 articles mentioned blinding the outcome evaluators, suggesting that they were in low risk; 1 article did not mention whether the outcome assessor was blinded, suggesting that the risk was unclear; and the last article did not clearly describe it, so it was high risk; (V) data completeness: the research data of 2 articles were complete, indicating low risk; (VI) selective reporting: 3 articles had no selective report, suggesting low risk; 1 article was unclear, because it was unclear risk; 1 article had selective report, which was high risk.; (VII) whether there were other biases: 1 article of high quality, so it was low risk; 1 article was impossible to determine whether there were other biases, which suggested that the risk was unclear; other articles were high risk.

\section{Incidence of atelectasis}

Five articles reported the incidence of atelectasis after surgery. The results of heterogeneity analysis showed that $\mathrm{I}^{2}=44 \%$ and $\mathrm{P}=0.13$, indicating that there was no obvious heterogeneity in the included articles, so the FEM was used for meta-analysis. The combined effect model analysis 
results showed that compared with the control group, the use of positive end expiratory pressure (PEEP) during mechanical ventilation can significantly reduce the incidence of atelectasis $(\mathrm{OR}=0.46 ; 95 \% \mathrm{CI}: 0.31-0.67 ; \mathrm{Z}=3.94$, $\mathrm{P}<0.0001)$. It is suggested that preoperative intervention can reduce the incidence of postoperative atelectasis in patients (as shown in Figure 4).

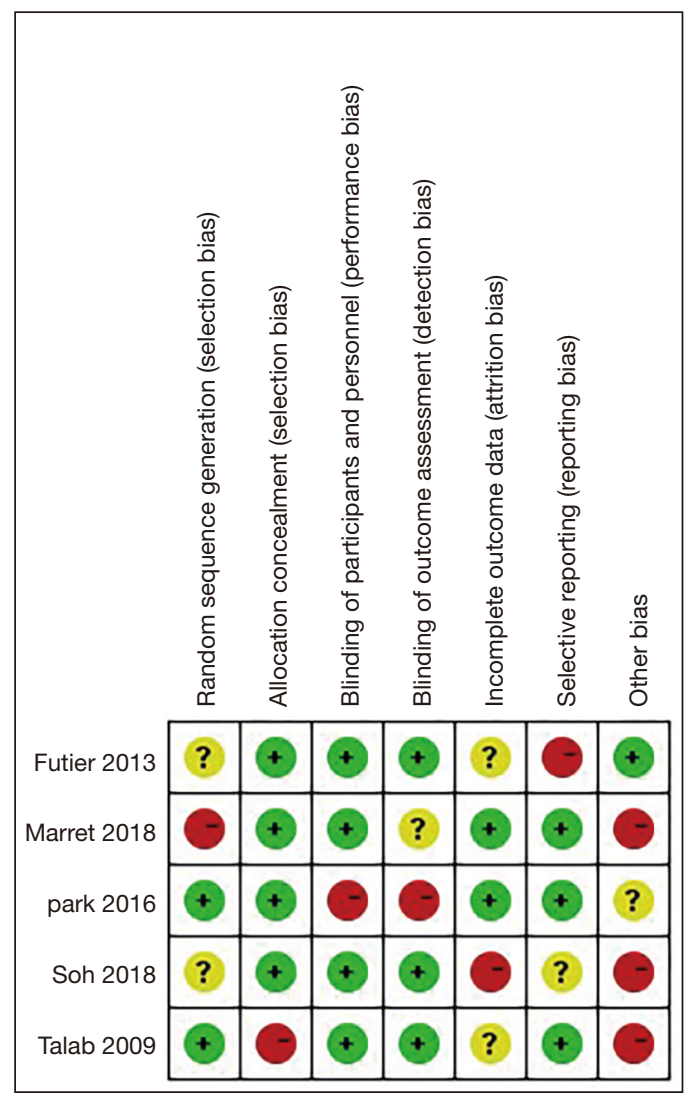

Figure 3 Multiple studies in the articles correspond to the multiple risk bias evaluation results.

\section{Analysis of publication bias}

Funnel chart analysis was performed on the 5 included articles, and the results showed that the funnel charts were asymmetric (Figures 5), indicating that there may have been publication bias. This may be related to factors such as the small sample size included in the study. However, it was within the $95 \% \mathrm{CI}$ as a whole, indicating that the article had high credibility. The postoperative pulmonary infection funnel chart was a bit scattered outside the credible interval, suggesting that the literature had publication bias.

\section{Discussion}

In this meta-analysis, there were a total of 5 articles (14-18) included. The limitations of interventional measures in this study indicated that participants and patients may have had measurement biases. In order to improve the reliability and reference of the research, it is expected that the research method and design could be further improved in the future. The use of preoperative interventions such as changing the dose of general anesthesia, continuous PEEP, non-invasive pressure support ventilation, and implementing physical therapy can reduce the incidence of PPC in thoracic surgery patients and improve the lung function of patients. Such results were consistent with the findings of Jakobsen et al. (19), which showed that preoperative general anesthesia and hypoventilation can reduce the resistance of patients during surgery. In 5 articles (14-18), there were slight differences in preoperative interventional methods, but all treatments had the effect of shortening the patient's blood loss, pleural expansion, and hospital stay. In addition, in the same study, the surgical method in the control group and the restorative drugs in the treatment group were the same. Therefore, the intervention of patients undergoing thoracic

\begin{tabular}{|c|c|c|c|c|c|c|c|c|c|c|}
\hline Study or Subgroup & \multicolumn{2}{|c|}{ Experimental } & \multicolumn{2}{|c|}{ Control } & Weight & $\begin{array}{c}\text { Odds Ratio } \\
\text { M-H, Fixed, } 95 \% \mathrm{Cl}\end{array}$ & \multicolumn{4}{|c|}{$\begin{array}{c}\text { Odds Ratio } \\
\text { M-H, Fixed, } 95 \% \mathrm{Cl}\end{array}$} \\
\hline Futier 2013 & 13 & 200 & 34 & 200 & $41.5 \%$ & $0.34[0.17,0.66]$ & & & & \\
\hline Marret 2018 & 20 & 172 & 27 & 171 & $31.3 \%$ & $0.70[0.38,1.31]$ & & & & \\
\hline park 2016 & 3 & 21 & 8 & 19 & $9.4 \%$ & $0.23[0.05,1.05]$ & & & & \\
\hline Soh 2018 & 2 & 39 & 0 & 39 & $0.6 \%$ & $5.27[0.24,113.35]$ & & & & \\
\hline Talab 2009 & 13 & 44 & 14 & 22 & $17.2 \%$ & $0.24[0.08,0.71]$ & & & & \\
\hline Total $(95 \% \mathrm{CI})$ & & 476 & & 451 & $100.0 \%$ & $0.46[0.31,0.67]$ & & & & \\
\hline Total events & 51 & & 83 & & & & & & & \\
\hline $\begin{array}{l}\text { Heterogeneity: } \mathrm{Chi}^{2}= \\
\text { Test for overall effect }\end{array}$ & $\begin{array}{l}7.17, \mathrm{df}= \\
Z=3.94\end{array}$ & $\begin{array}{l}(P=0 . \\
<0.000\end{array}$ & $\begin{array}{l}\text { 13); }\left.\right|^{2}=4 \\
01)\end{array}$ & $44 \%$ & & & 0.01 & [experimental] & 10 & 100 \\
\hline
\end{tabular}

Figure 4 Forest map for incidence of atelectasis. CI, confidence interval. 


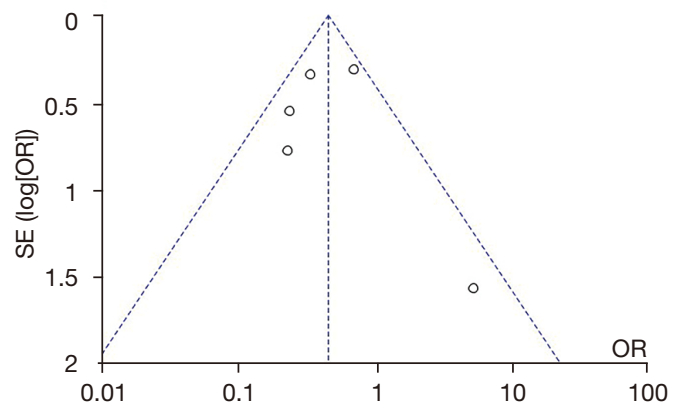

Figure 5 The funnel chart for bias of publication of the included articles in terms of atelectasis index for patients with thoracic surgery. SE, standard error; OR, odds ratio.

surgery improved lung function and reduced the incidence of postoperative lung complications $(20,21)$. For patients undergoing general anesthesia, mechanical ventilation is used during the operation. Regardless of whether there is lung disease before anesthesia, about $75 \%$ of patients are in a state of local alveoli in the process of general anesthesia and mechanical ventilation. This situation can directly lead to the lungs. Increased shunt induce hypoxemia in severe cases. Preoperative intervention can reduce the incidence of postoperative atelectasis. The reason may be the clinically commonly used volume control ventilation mode that can't fully open the alveoli and excessive tidal volume. Not only the effect of improving the oxygenation status is poor, but there is a risk of pneumothorax. The low level of PEEP makes the alveoli and small bronchi in an open state, thereby increasing the number of effective ventilation alveoli, avoiding unnecessary exudation of the alveoli, improving the oxygen-carrying function of lung hemoglobin, and increasing $\mathrm{PaO}_{2}$, and fundamentally acting hypoxemia (22).

It is necessary to study whether preoperative and postoperative intervention can reduce PPC in patients undergoing thoracic surgery, and further investigate the specific time of preoperative intervention. In addition, it is imperative to avoid wasting medical resources and reduce PPC. The safety of intervention measures such as ventilation in thoracic surgery has also been widely recognized. The British Thoracic Association pointed out that the use of ventilation intervention is safe and effective in the diagnosis and treatment of patients in the intensive care unit, the elderly, and heart disease. Pulmonary infection and atelectasis prevention plan and control measures are as follows: (I) reduce or eliminate the colonization and inhalation of pathogenic bacteria in the oropharynx and gastrointestinal tract to prevent the occurrence of endogenous infections; (II) strengthen the nursing of endotracheal intubation or tracheotomy, and correctly master the sputum suction operation. Foreign countries use special endotracheal cannula devices to prevent the inhalation of oropharyngeal bacteria and reduce ventilatorassociated pneumonia by $50 \%$. For high-risk and susceptible patients, selective digestive tract decontamination (SDD) is used to kill pathogenic bacteria in the gastrointestinal tract by applying antibacterial drugs that are not absorbed by the gastrointestinal tract to avoid their migration or translocation. Commonly used antibacterial drugs are tobramycin, polymyxin $\mathrm{E}$, and amphotericin $\mathrm{B}$.

In this study, the patient's breathing, preoperative evaluation, or the effect of preoperative inhalation of a certain drug were studied. Since there was no comparison between the perioperative control group and the treatment group, the relevant Chinese articles were not included. In addition, the treatment times of the 8 included articles in this study were contradictory, which may have a certain impact on the results of this study. There was no report on the randomization method or allocation concealment. Therefore, it is recommended to further improve the experimental plan, standardize the specific time, methods, and drugs of periodic intervention, and implement highquality, large-scale samples. Additionally, multi-center randomized controlled tests need to be performed to obtain more reliable evidence.

\section{Conclusions}

The results of this study analyzed the interventions (such as changing the dose of general anesthesia, continuous PEEP, non-invasive pressure support ventilation, and implementation of physical therapy) in perioperative circumstances that included comparative thoracic surgery (such as pulmonary lobectomy, thoracic surgery, and lung cancer surgery) in the treatment of pulmonary infection and atelectasis. In addition, the intervention method was convenient and easy to operate. Therefore, more highquality, large-sample, multi-center randomized controlled studies should be carried out clinically, and after more reliable evidence was obtained, they should be promoted and applied clinically.

\section{Acknowledgments}

Funding: None. 


\section{Footnote}

Reporting Checklist: The authors have completed the PRISMA reporting checklist. Available at https://dx.doi. org/10.21037/apm-21-2441

Conflicts of Interest: All authors have completed the ICMJE uniform disclosure form (available at https://dx.doi. org/10.21037/apm-21-2441). The authors have no conflicts of interest to declare.

Ethical Statement: The authors are accountable for all aspects of the work in ensuring that questions related to the accuracy or integrity of any part of the work are appropriately investigated and resolved.

Open Access Statement: This is an Open Access article distributed in accordance with the Creative Commons Attribution-NonCommercial-NoDerivs 4.0 International License (CC BY-NC-ND 4.0), which permits the noncommercial replication and distribution of the article with the strict proviso that no changes or edits are made and the original work is properly cited (including links to both the formal publication through the relevant DOI and the license). See: https://creativecommons.org/licenses/by-nc-nd/4.0/.

\section{References}

1. Ceppa DP, Kosinski AS, Berry MF, et al. Thoracoscopic lobectomy has increasing benefit in patients with poor pulmonary function: a Society of Thoracic Surgeons Database analysis. Ann Surg 2012;256:487-93.

2. Nagasaki F, Flehinger BJ, Martini N. Complications of surgery in the treatment of carcinoma of the lung. Chest 1982;82:25-9.

3. Canet J, Gallart L, Gomar C, et al. Prediction of postoperative pulmonary complications in a populationbased surgical cohort. Anesthesiology 2010;113:1338-50.

4. Khuri SF, Henderson WG, DePalma RG, et al. Determinants of long-term survival after major surgery and the adverse effect of postoperative complications. Ann Surg 2005;242:326-41; discussion 341-3.

5. Fernandez-Bustamante A, Frendl G, Sprung J, et al. Postoperative pulmonary complications, early mortality, and hospital stay following noncardiothoracic surgery: a multicenter study by the perioperative research network investigators. JAMA Surg 2017;152:157-66.

6. Miskovic A, Lumb AB. Postoperative pulmonary complications. Br J Anaesth 2017;118:317-34.

7. Shander A, Fleisher LA, Barie PS, et al. Clinical and economic burden of postoperative pulmonary complications: patient safety summit on definition, riskreducing interventions, and preventive strategies. Crit Care Med 2011;39:2163-72.

8. Lawrence VA, Cornell JE, Smetana GW, et al. Strategies to reduce postoperative pulmonary complications after noncardiothoracic surgery: systematic review for the American College of Physicians. Ann Intern Med 2006;144:596-608.

9. Fleisher LA, Fleischmann KE, Auerbach AD, et al. 2014 ACC/AHA guideline on perioperative cardiovascular evaluation and management of patients undergoing noncardiac surgery: executive summary: a report of the American College of Cardiology/American Heart Association Task Force on Practice Guidelines. Circulation 2014;130:2215-45.

10. Moher D, Liberati A, Tetzlaff J, et al. Preferred reporting items for systematic reviews and meta-analyses: the PRISMA statement. PLoS Med 2009;6:e1000097.

11. Östberg E, Thorisson A, Enlund M, et al. Positive endexpiratory pressure and postoperative atelectasis: a randomized controlled trial. Anesthesiology 2019;131:809-17.

12. Pereira SM, Tucci MR, Morais CCA, et al. Individual positive end-expiratory pressure settings optimize intraoperative mechanical ventilation and reduce postoperative atelectasis. Anesthesiology 2018;129:1070-81.

13. Pehlivan E, Turna A, Gurses A, et al. The effects of preoperative short-term intense physical therapy in lung cancer patients: a randomized controlled trial. Ann Thorac Cardiovasc Surg 2011;17:461-8.

14. Marret E, Cinotti R, Berard L, et al. Protective ventilation during anaesthesia reduces major postoperative complications after lung cancer surgery: a doubleblind randomised controlled trial. Eur J Anaesthesiol 2018;35:727-35.

15. Futier E, Constantin JM, Paugam-Burtz C, et al. A trial of intraoperative low-tidal-volume ventilation in abdominal surgery. N Engl J Med 2013;369:428-37.

16. Talab HF, Zabani IA, Abdelrahman HS, et al. Intraoperative ventilatory strategies for prevention of pulmonary atelectasis in obese patients undergoing laparoscopic bariatric surgery. Anesth Analg 2009;109:1511-6.

17. Park SJ, Kim BG, Oh AH, et al. Effects of intraoperative protective lung ventilation on postoperative pulmonary complications in patients with laparoscopic surgery: 
prospective, randomized and controlled trial. Surg Endosc 2016;30:4598-606.

18. Soh S, Shim JK, Ha Y, et al. Ventilation with high or low tidal volume with PEEP does not influence lung function after spinal surgery in prone position: a randomized controlled trial. J Neurosurg Anesthesiol 2018;30:237-45.

19. Jakobsen JC, Wetterslev J, Winkel P, et al. Thresholds for statistical and clinical significance in systematic reviews with meta-analytic methods. BMC Med Res Methodol 2014;14:120.

20. Futier E, Paugam-Burtz C, Godet T, et al. Effect of early postextubation high-flow nasal cannula vs conventional oxygen therapy on hypoxaemia in patients after major

Cite this article as: Zhao $\mathrm{Y}$, Zheng $\mathrm{R}$, Xiang W, Ning D, $\mathrm{Li} Z$. Systematic review and meta-analysis on perioperative intervention to prevent postoperative atelectasis complications after thoracic surgery. Ann Palliat Med 2021;10(10):10726-10734. doi: 10.21037/apm-21-2441 abdominal surgery: a French multicentre randomised controlled trial (OPERA). Intensive Care Med 2016;42:1888-98.

21. Wetterslev J, Thorlund K, Brok J, et al. Trial sequential analysis may establish when firm evidence is reached in cumulative meta-analysis. J Clin Epidemiol 2008;61:64-75.

22. Kindgen-Milles D, Müller E, Buhl R, et al. Nasalcontinuous positive airway pressure reduces pulmonary morbidity and length of hospital stay following thoracoabdominal aortic surgery. Chest 2005;128:821-8.

(English Language Editor: J. Jones) 